\title{
Atypical facial onset Guillain-Barré syndrome following first dose of COVISHIELD vaccine
}

\author{
R.V. Aravinda' ${ }^{1}$, Priyanka Mahendra Tater ${ }^{2}$, Harsha Huliyappa ${ }^{1}$, Christy Joseph Manual' \\ ${ }^{1}$ Narayana Multispeciality Hospital, Mysore, India \\ 2 Sir HN Reliance Foundation Hospital and Research Centre, Mumbai, India
}

\begin{abstract}
In the midst of the global pandemic of COVID-19 and its significant morbidity and mortality reported across the world due to severe acute respiratory distress syndrome (SARS), it has always been posing a new set of complications each passing day. As we are still in the process of understanding about the complications related to COVID-19, we are encountered with complications related to immunization for COVID-19. We are reporting a case of facial onset Guillain-Barré syndrome (GBS) in the patient who received first dose of COVISHIELD vaccine a couple of weeks prior to the onset of his illness.
\end{abstract}

Keywords: atypical GBS, COVISHIELD vaccine, COVID-19

\section{INTRODUCTION}

After its initial recognition in Wuhan, China, the COVID-19 took little time in becoming a global pandemic taking lives of thousands of people across the world (1). Apart from its primary presentation involving the respiratory system ranging from mild flu like symptoms to severe acute respiratory distress syndrome (SARS), COVID-19 infection has also shown its effects on various other systems of the body. Of importance is the involvement of nervous system. Several cases of encephalitis, stroke and peripheral nervous system disorders are reported including GBS following the infection (2).

With the limited current understanding of the disease, considering the catastrophic health and economic consequences of the COVID-19, political and health-care leaders worldwide are looking at the vaccination campaigns aggressively with a hope to mitigate the problem. The vaccination too, has shown to have its own complications ranging from injection site pain, myalgia, sore throat, fever, anaphylactic reactions, Bell's palsy (3) and even GBS (4). However, atypical form of GBS with the facial onset following the first dose of COVISHIELD vaccine has not been reported to date (April 23, 2021).

\section{CASE PRESENTATION}

A 73-year-old gentleman who had received his first dose of COVISHIELD vaccine on $22^{\text {nd }}$ of March 2021 (fig. 1) developed mild deviation of angle of mouth to the right and subtle slurring of speech in the evening of $4^{\text {th }}$ April, 2021. He had the history of diabetes mellitus for the last 4-5 years. He did not seek medical attention urgently and retired that night. After he woke up the following day he noticed significant difficulty of speech, inability to approximate both the lips tightly to each other and inability to close both the eyes tightly. He consulted the primary care physician the next day and underwent an plain MRI of brain to rule out stroke. MRI brain did not reveal any acute stroke (fig. 2). He was then referred to the neurosciences division of our hospital for further evaluation and management. On arrival to our facility ( $5^{\text {th }}$ day of the onset of illness), his physical examination revealed normal mental status; cranial nervous system examination re- 
vealed bifacial weakness to an extent of inability to close the eyes and approximate the lips without other cranial nerve involvement. Motor examination of limbs did not show any weakness. However, his deep tendon reflexes in all the limbs were diminished. Examination for co-ordination was normal. Even though he could walk without assistance, he could not perform tandem gait. His sensory examination was normal. There were no signs of meningeal irritation.

Considering the predominant deficit bilateral facial weakness, he was further evaluated for bilateral facial palsy. He had a history of poorly controlled diabetes with the blood sugar level at the the time of presentation being $323 \mathrm{mg} / \mathrm{dl}$. He underwent contrast enhanced MRI brain (fig. 3), that revealed a granuloma in the right basifrontal region. However, the lesion was asymptomatic. Nerve conduction study of the facial nerves and the limbs was done and that showed relatively lower amplitude compound muscle action potentials (CMAPs) of both sides of the face. There was prolongation of the distal latencies of the median and ulnar nerves with mild reduction in the CMAP amplitudes.

By the next morning he developed weakness of both the lower limbs and was unable to walk even with assistance. His motor power was reduced to 4-/5 in both the lower limbs. CSF analysis was done and it showed cell count of $3 /$ cumm and protein of $136 \mathrm{mg} / \mathrm{dl}$ which was suggestive of albumino-cytological dissociation, a characteristic feature in GBS. He was treated with intravenous immunoglobulin (IVIg) therapy and was kept under close monitoring. His motor power in both the lower limbs reduced to 1/5 in the next 3-4 days, the power in left upper limb reduced to $3 / 5$ and in right upper limb reduced to $4 / 5$ concurrently. A repeat nerve conduction study was performed at this time and it revealed further prolongation of distal latencies and reduction in CMAP amplitudes. His motor functions remained as such for the next 2-3 days then he started to show improvement in his facial muscles. He was able to close his eyes by the next couple of days and the left upper limb power improved to 4-/5. Meanwhile serum electrolyte panel was being monitored anticipating hyponatremia which he developed after 4 days of admission to our hospital. Initially, oral sodium supplementation was given which did not improve his serum sodium levels and hence intravenous 3\% sodium chloride infusion was started. In the next few days his serum sodium level stabilized and his motor functions improved further.

He was concurrently evaluated for the possible cause of right basifrontal granuloma; no specific etiology could be identified so far. Since it was asymptomatic and all essential work up was negative, he was asked for a repeat imaging during follow up. He was discharged after 15 days of hospital stay.

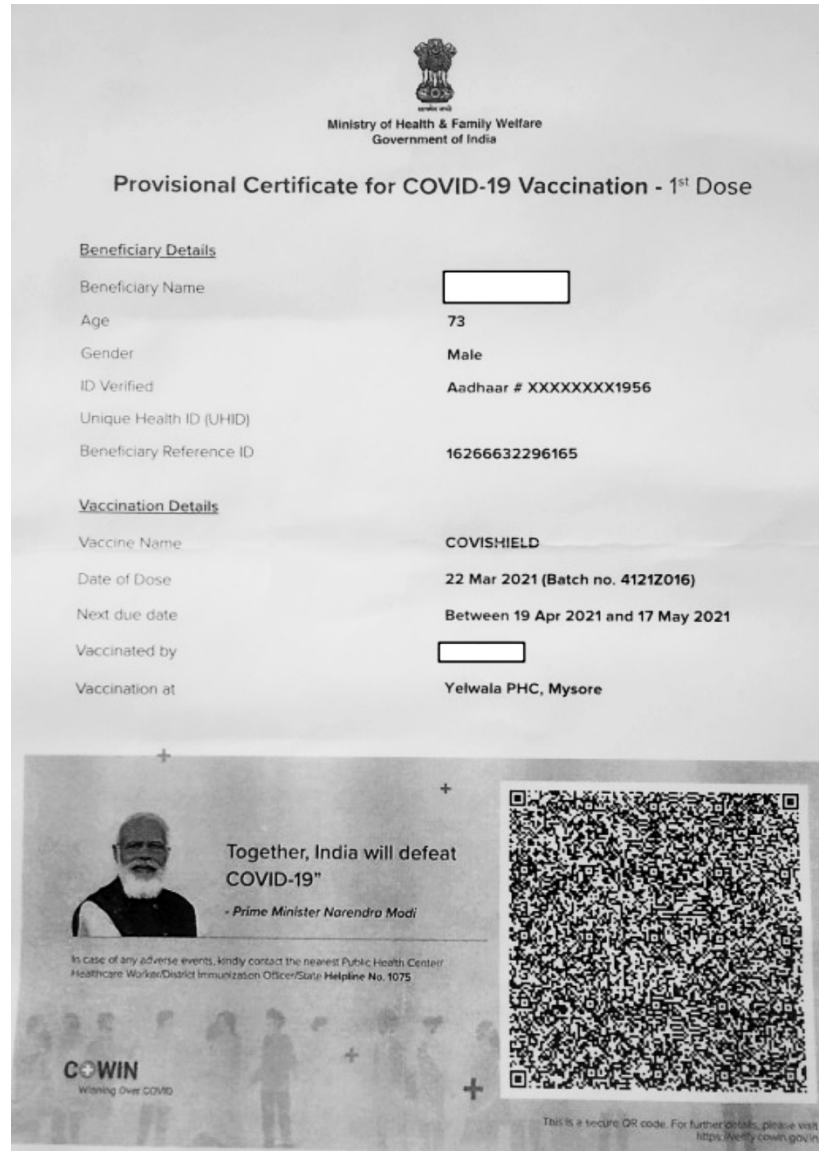

FIGURE 1. Provisional certificate issued to the patient after his $1^{\text {st }}$ dose of vaccination

\section{DISCUSSION}

GBS is acute inflammatory polyradiculoneuropathy which can be preceded by several viral or bacterial infections. It can also develop following certain vaccinations (5). Even though the exact mechanism is unclear, autoimmunity is thought to play a role in GBS (6). It is one of the common causes of acute flaccid paralysis world-wide. Usually, it presents with ascending weakness starting from the lower limbs. Atypical form of GBS such as cervico-brachial onset, Miller Fisher syndrome are also well reported (7). In most of the cases the weakness progresses for the first two weeks and then stabilizes. During this progression, about one third of the patients develop respiratory failure and may need ICU admission for assisted ventilation. Most of the patients recover reasonably well particularly if intervened in the initial days of the illness. IVIg or plasma exchange therapies are widely used in the management of GBS and both have comparable response rates. However, IVIg is largely preferred over plasma exchange owing to its ease of administration and safety profile.

Since the outbreak of the COVID-19 pandemic world-wide there has been a vigorous hunt for the vaccine to curtail the further spread. ChAdOx1 nCoV- 19 coronavirus vaccine (recombinant) or 


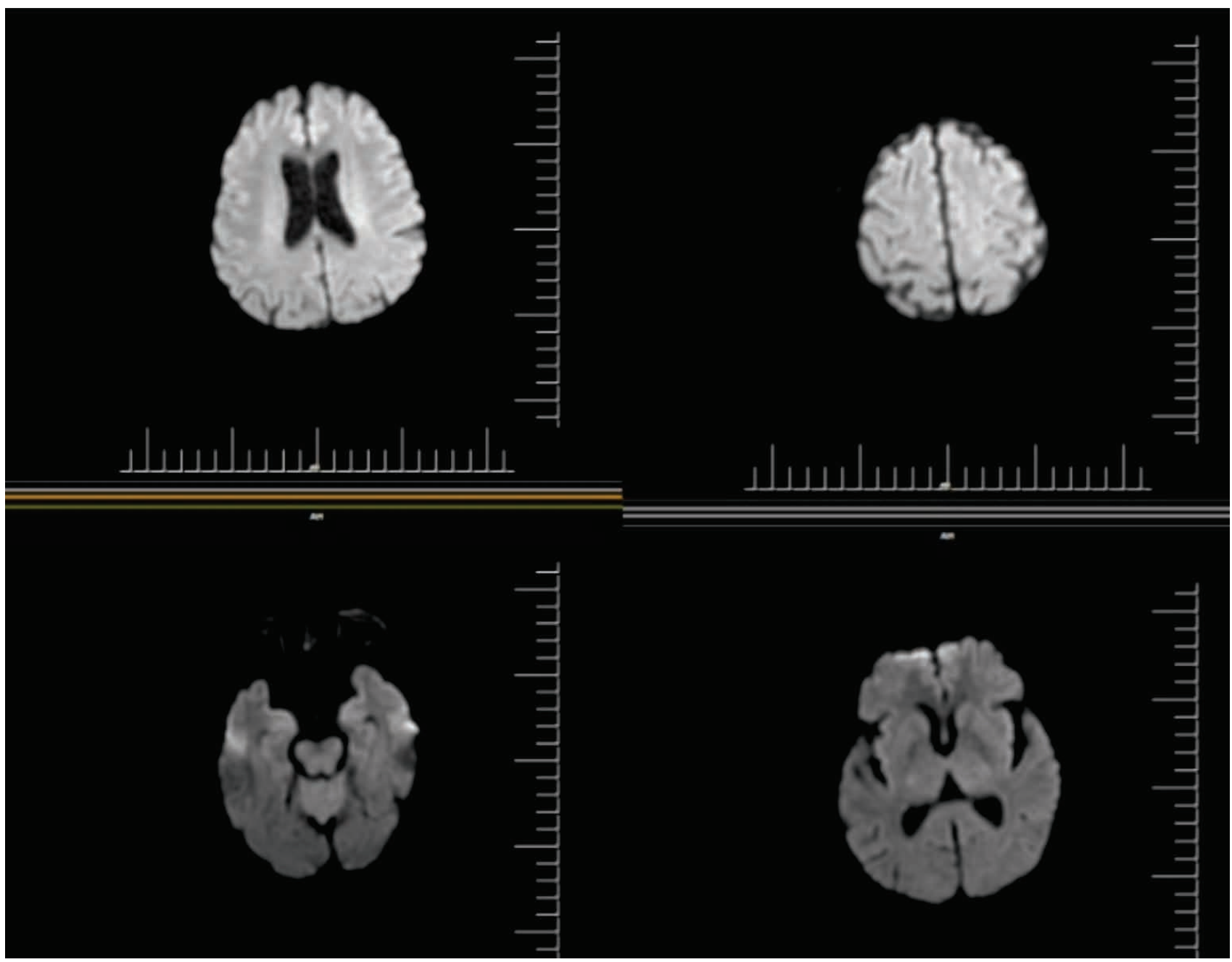

FIGURE 2. DWI-MRI brain of the patient showing no acute infarct

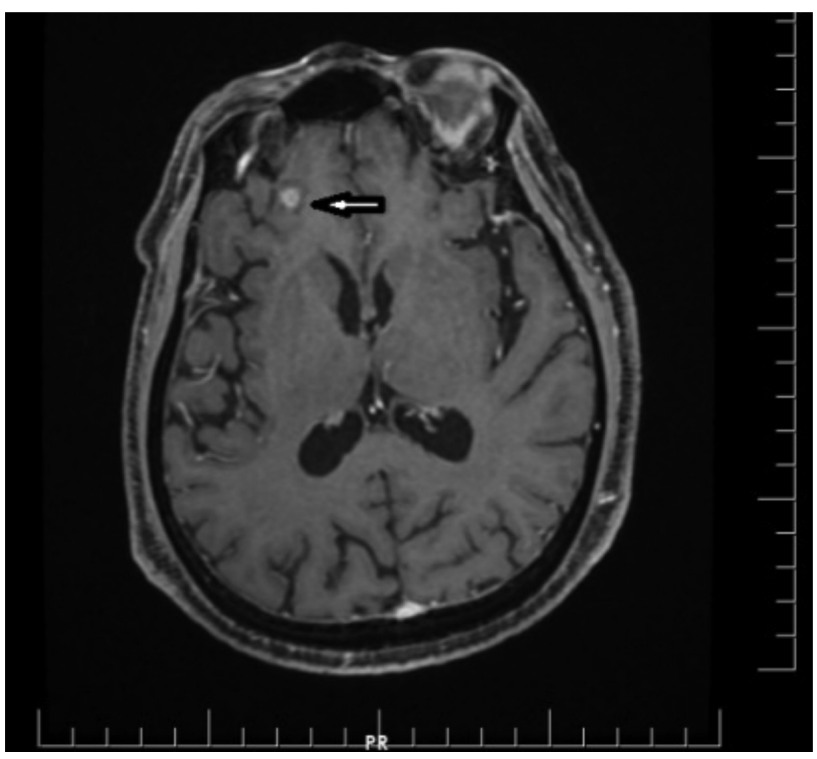

FIGURE 3. Contrast enhanced MRI of brain showing right basifrontal granuloma

COVISHIELD is one such vaccine widely used in India and other countries as the part of a massive vaccination campaign. This active vaccination is given in two separate doses, 4-6 weeks apart. It is given as
$0.5 \mathrm{ml}$ intramuscular injections in the deltoid. It is a monovalent vaccine composed of a single recombinant, replication-deficient chimpanzee adenovirus (ChAdOx1) vector encoding the $S$ glycoprotein of SARS-CoV-2. Following administration, the S glycoprotein of SARS-CoV-2 is expressed locally stimulating neutralizing antibody and cellular immune responses (8). This immune response may at times trigger autoimmunity against the myelin and may result into GBS.

\section{CONCLUSIONS}

The incidence of atypical-facial onset GBS is rare. We report the first case of facial onset GBS after the first dose of COVISHIELD vaccine in India. With the mass vaccination campaign on the way world-wide, it is imperative for the clinicians to be aware of the possible complications of the vaccines; their identification at the earliest and management is far more crucial. More research is warranted for better understanding of the pathogenesis of GBS, particularly in the post vaccination setting. Major neurological complications following vaccination is rare and the threat of such complications alone should not be 
taken into consideration in the decision making for the vaccination. We encourage to adhere to the guidelines provided by the concerned national and

\section{REFERENCES}

1. Lu H, Stratton CW, Tang YW. Outbreak of pneumonia of unknown etiology in Wuhan, China: The mystery and the miracle. J Med Virol. 2020;92:401-2.

2. Taherifard E. Neurological complications of COVID-19: a systematic review. Neurol Res. 2020 Nov;42(11):905-912.

3. Colella G, Orlandi M, Cirillo N. Bell's palsy following COVID-19 vaccination. J Neurol. 2021;1-3.

4. Goss AL, Samudralwar RD, Das RR, Nath A. ANA Investigates: Neurological Complications of COVID-19 Vaccines. Ann Neurol. 2021 May;89(5):856-857.

5. Haber P, Sejvar J, Mikaeloff Y, DeStefano F. Vaccines and Guillain-Barré syndrome. Drug Saf. 2009;32(4):309-23. international authorities regarding the management of COVID-19 and related illnesses/complications.

\section{Conflict of interest: none declared} Financial support: none declared

6. Israeli E, Agmon-Levin N, Blank M, Chapman J, Shoenfeld Y. Guillain-Barré syndrome - a classical autoimmune disease triggered by infection or vaccination. Clin Rev Allergy Immunol. 2012 Apr;42(2):121-30.

7. Pradhan RR, Yadav SK, Yadav SK Sr. Pharyngeal-Cervical-Brachial variant of Guillian-Barré Syndrome in Children. Cureus. 2020;12(2):e6983.

8. Covishield_ChAdOx1_nCoV19_corona_virus_vaccine_insert. Available at: www.seruminstitute.com. 